TRANSACTIONS OF THE

AMERICAN MATHEMATICAL SOCIETY

Volume 177, March 1973

\title{
REDUCTIONS OF IDEALS IN COMMUTATIVE RINGS
}

\author{
BY
}

\section{JAMES H. HAYS}

ABSTRACT. All rings considered in this paper are commutative, associative, and have an identity. If $A$ and $B$ are ideals in a ring, then $B$ is a reduction of $A$ if $B \subseteq A$ and if $B A^{n}=A^{n+1}$ for some positive integer $n$. An ideal is basic if it has no reductions. These definitions were considered in local rings by Northcott and Rees; this paper considers them in more general rings. Basic ideals in Noetherian rings are characterized to the extent that they are characterized in local rings. It is shown that elements of the principal class generate a basic ideal in a Noetherian ring. Prüfer domains do not have the basic ideal property, that is, there may exist ideals which are not basic; however, a characterization of Prüfer domains can be given in terms of basic ideals. A domain is Prüfer if and only if every finitely generated ideal is basic.

1. Introduction. All rings considered in this paper are commutative, associative and have an identity. We are concerned with the following definitions, which appeared originally in a paper by Northcott and Rees [6].

Definition 1.1. If $A$ and $B$ are ideals of a ring $R$, then $B$ is a reduction of $A$ if $B \subseteq A$ and $B A^{n}=A^{n+1}$ for at least one positive integer $n$.

Definition 1.2. An ideal which has no reductions other than itself is called a basic ideal.

In [6], Northcott and Rees considered reductions of ideals in a local ring with an infinite residue field; and in a second paper [7] they considered the same problem for equicharacteristic local rings.

In this paper, we investigate reductions of ideals and basic ideals in more general rings. We give several sufficient conditions in order that an ideal be basic; for example, in a Noetherian ring elements of the principal class generate a basic ideal.

We note in $\$ 2$ that the product of basic ideals need not be basic, but in $\S 3$ we give two sufficient conditions in order that such a product be basic. The most important theorem in $\$ 3$ is a characterization of basic ideals in Noetherian rings: An ideal $A$ in a Noetherian ring $R$ is basic if and only if $A R_{M}$ is basic for each

Presented to the Society, January 18, 1972; received by the editors July 22, 1971. AMS (MOS) subject classifications (1970). Primary 13A15; Secondary 13F05,13C05.

Key words and phrases. Reductions of ideals, basic, Noetherian rings, Prüfer domains, valuation, valuation ring, $C$-ideals, analytically independent, principal class, Dedekind domains. 
maximal ideal $M$ of $R$. This result characterizes basic ideals in Noetherian rings to the extent that they are characterized in local rings.

In $\$ 5$ we define $C$-ideals and then obtain some simple results about them. We note that in the case of a Noetherian ring, $C$-ideals can be identified with integrally complete ideals; however, these definitions are not equivalent.

$\$ 6$ is devoted to results concerning valuation rings and Prüfer domains, the most important of which is that a domain is Prüfer if and only if every finitely generated ideal is basic. We also show that Prüfer domains need not have the basic ideal property; that is, there may exist ideals which are not basic. We exhibit such an ideal in a valuation ring and, by this example, observe that several results about reductions of ideals in Noetherian rings do not extend to non-Noetherian rings. This example also establishes the existence of an ideal that has no minimal reductions.

We will frequently refer to the following well-known result.

Lemma 1.3 [4, Theorem 3.10, p. 42]. Let $A$ be an ideal of the ring $R$. Let $\left\{M_{\boldsymbol{\lambda}}\right\}_{\boldsymbol{\lambda} \in \mathbf{\Lambda}}$ be the set of maximal ideals of $R$, and for each $\lambda$ let $e_{\boldsymbol{\lambda}}, c_{\boldsymbol{\lambda}}$ denote extension and contraction of ideals with respect to the quotient ring $R_{M_{\lambda}} \cdot$ If $\Sigma$ is the set of maximal ideals that contain $A$, then

(1) $A=\bigcap_{\boldsymbol{\lambda} \in \mathbf{\Lambda}} A^{e_{\lambda} c_{\lambda}}$, and

(2) $A=\bigcap_{\sigma \in \boldsymbol{\Sigma}} A^{\boldsymbol{e}_{\sigma} c_{\sigma}}$.

If $R$ is an integral domain, then

(3) $A=\bigcap_{\boldsymbol{\lambda} \in \mathbf{\Lambda}} A^{e} \boldsymbol{\lambda}$.

We will list at this time the results from [6] that are pertinent to this paper and which indicate the nature of the problem.

Let $v_{1}, v_{2}, \cdots, v_{r}$ be elements of a local ring $(Q, M)$. The elements $v_{1}, v_{2}$, $\cdots, v_{r}$ are analytically independent if for each form $\phi\left(X_{1}, \ldots, X_{r}\right)$ with coefficients in $Q$ such that $\phi\left(v_{i}\right)=0$, it follows that all the coefficients of $\phi$ are in $M$.

Theorem $1.4[6$, Theorem 4, p. 152]. Let $(Q, M)$ be a local ring such that $Q / M$ is infinite. An ideal $A$ is basic if and only if it admits an analytically independent generating set.

The next two theorems are proved in [6] for an ideal $A$ in a local ring $(Q, M)$, such that $Q / M$ is infinite, but an examination of the proofs of these theorems shows that they hold for arbitrary Noetherian rings. An ideal which does not consist entirely of zero divisors is called a regular ideal.

Theorem 1.5. Let $A$ be an ideal in a Noetherian ring. Then

(a) $[6$, Theorem $1, \mathrm{p} .155]$. There exists a unique ideal, $\hat{A}$, with the following two properties: 
(1) $A$ is a reduction of $\hat{A}$.

(2) Every ideal which bas $A$ as a reduction is contained in $\hat{A}$.

(b) $\left[6\right.$, Theorem 3, p. 156]. If $A$ is regular, then $\hat{A}=\left\{x \mid x^{n}+a_{1} x^{n-1}+\cdots+\right.$ $a_{n}=0$ where $\left.a_{i} \in A^{i}\right\}$.

2. Elements of the principal class. We noted in Theorem 1.4 that, in the local rings studied in [6], basic ideals are characterized as those that can be generated by analytically independent elements. It is of interest to see how this theorem can be extended to Noetherian rings, and to this end we first extend the notion of analytic independence to Noetherian rings.

One such generalization is given by Davis [2]. In order to avoid confusion, in the following definition we will say that " $x_{1}, \ldots, x_{n}$ are of the principal class," instead of the terminology used by Davis " $x_{1}, \ldots, x_{n}$ are analytically independent".

Definition 2.1. Let $x_{1}, \cdots, x_{n}$ be elements of a Noetherian ring. The elements $x_{1}, \ldots, x_{n}$ are of the principal class if for each form $\phi\left(X_{1}, \ldots, X_{n}\right)$ such that $\phi\left(x_{i}\right)=0$, it follows that all the coefficients of $\phi$ are in $\operatorname{rad}\left(x_{1}, \ldots, x_{n}\right)$.

By the beight of an ideal in a Noetherian ring, we mean the minimum of the heights of the associated primes. An ideal is of the principal class if it has height $n$ and can be generated by $n$ elements.

Davis has shown ([2, Corollary 1, p. 199] and [3, Proposition 1, p. 49]) that an ideal $A$ in a Noetherian ring is of the principal class if and only if $A$ can be generated by elements of the principal class.

We will show that if $x_{1}, \ldots, x_{n}$ are of the principal class, then the ideal $\left(x_{1}, \cdots, x_{n}\right)$ is basic; we will then give an example to show that the converse is not true.

The following lemma, which provides a sufficient condition for an ideal to be basic in any ring, is needed here.

Lemma 2.2. Let $A$ be an ideal in a ring $R$ and let $\left\{M_{\lambda}\right\}$ be the set of maximal ideals of $R$. Let $e_{\lambda}$ denote the extension of ideals to $R_{M_{\lambda}}$ and let $c_{\lambda}$ denote the contraction of ideals of $R_{M_{\lambda}}$ to $R$. If $A^{e} \lambda$ is basic for each $\lambda$, then $A$ is basic.

Proof. Let $B$ be a reduction of $A$; then $B^{e} \lambda$ is a reduction of $A^{e} \lambda$ for each $\lambda$. Since $A^{e_{\lambda}}$ is basic, $A^{e_{\lambda}}=B^{{ }^{e} \lambda}$ for each $\lambda$. Then $B=\bigcap_{\lambda} B^{{ }^{e}{ }_{\lambda}{ }^{c} \lambda}=\bigcap_{\lambda} A^{e_{\lambda}{ }^{c} \lambda}$ $=A$ by Lemma 1.3 .

We will use the notation $e_{\lambda}$ and $c_{\lambda}$ as in Lemma 2.2 for the remainder of the paper.

Theorem 2.3. If $R$ is Noetherian and if the elements $x_{1}, \cdots, x_{n}$ are of the principal class, then the ideal generated by $\left\{x_{i}\right\}_{i=1}^{n}$ is basic. 
Proof. We show first that $\left\{\phi\left(x_{1}\right), \ldots, \phi\left(x_{n}\right)\right\}$ is an analytically independent set in $R_{M_{\lambda}}$, where $M_{\lambda}$ is a maximal ideal containing $A$ and $\phi$ is the canonical homomorphism used in the formation of $R_{M_{\lambda}}$.

Suppose that $\left\{\phi\left(x_{1}\right), \ldots, \phi\left(x_{n}\right)\right\}$ is not analytically independent in $R_{M_{\lambda}}$. There exists a form $F\left(X_{1}, \ldots, X_{n}\right)^{n}$, with coefficients $\phi\left(a_{i}\right) / \phi\left(s_{i}\right)$ in $R_{M_{\lambda}}$ such that some $\phi\left(a_{i}\right) / \phi\left(s_{i}\right)$ is not in $M_{\lambda} R_{M_{\lambda}}$, and such that $F\left[\phi\left(x_{1}\right), \ldots, \phi\left(x_{n}\right)\right]=0$. Clearing this equation of fractions, we find that there exists a form $f\left(X_{1}, \ldots, X_{n}\right)$ with coefficients in $R$, at least one coefficient not in $M_{\lambda}$, and with the property that $\phi\left(f\left(x_{1}, \cdots, x_{n}\right)\right)=0$. By the definition of $\phi$, there exists an $m$ in $R-M_{\lambda}$ such that $m\left(f\left(x_{i}\right)\right)=0$. This is a contradiction of the fact that the elements $x_{i}$ are of the principal class.

We now need only show that in a local ring, analytically independent elements generate a basic ideal and then apply Lemma 2.2. That this is the case follows easily from [6, Lemmas 1 and 3, pp. 149 and 147], which do not depend on the special conditions concerning the residue field.

Corollary 2.4. An ideal in a Noetherian ring wbich is of the principal class is basic.

Lemma 2.5. Let $A$ be an ideal of a Noetherian ring which is of the principal class; then $A$ is principal or some power of $A$ is not basic.

Proof. There exist elements $x_{1}, \cdots, x_{r}$, which are of the principal class, such that $A=\left(x_{1}, \cdots, x_{r}\right)$. If $r=1$, the result holds. If $r \geq 2$, we claim that $\left(x_{1}^{r}, x_{2}^{r}, \cdots, x_{r}^{r}\right)$ . $\left(A^{r}\right)^{r-1}=\left(A^{r}\right)^{r}$. The ideal $\left(A^{r}\right)^{r}$ is generated by elements of the form $x_{1}^{i_{1}} \cdots x_{r}^{i_{r}}$, where $\Sigma_{j=1}^{r} i_{j}=r^{2}$. If $i_{j}<r$ for all $j$ then $\sum_{i=1}^{r} i_{i_{i}} \leq r(r-1)<(r)^{2}$. Thus $i_{j} \geq r$ for some $j$ and $x_{1}^{i} \ldots x_{r}^{i_{r}}=x_{j}^{r}\left(x_{1}^{i_{1}} \ldots x_{j}^{i_{j}-r^{j}=1} \cdots x_{r}^{i_{r}}\right) \in\left(x_{1}^{r}, \ldots, x_{r}^{r}\right)\left(A^{r}\right)^{r-1}$. We thus obtain $\left(A^{r}\right)^{r} \subseteq\left(x_{1}^{r}, \ldots, x_{r}^{r}\right)\left(A^{r}\right)^{r-1}$, and consequently $\left(x_{1}^{r}, \ldots, x_{r}^{r}\right)$ is a reduction of $A^{r}$. That this is a proper reduction follows from the fact that the elements $x_{i}$ are of the principal class.

Example 2.6. Let $A$ be a nonprincipal ideal in a Dedekind domain $D$. Then $A$ is invertible, hence basic, and can be generated by two elements $x, y$. By Lemma 2.5, the elements $x, y$ are not of the principal class.

Theorem 2.7. If $R$ is a Noetberian ring sucb that powers of basic ideals are basic, then the dimension of $R$ is $\leq 1$.

Proof. Suppose that $P$ is a prime ideal of height $n>1$. There exist $n$ elements $x_{1}, \ldots, x_{n}$ contained in $P$ that are of the principal class [8, Theorem 31, p. 242]. The ideal $A$ generated by these elements is basic by Theorem 2.3, and each power of $A$ is basic by hypothesis, a contradiction to Lemma 2.5 .

An ideal $A$ is said to be a cancellation ideal if the equation $A B=A C$, where $B$ and $C$ are ideals, implies that $B=C$. It is obvious that cancellation ideals 
are basic and that the product of cancellation ideals is a cancellation ideal. The following example shows that a basic ideal need not be a cancellation ideal.

Example 2.8. Let $R=k\left[X_{1}, \ldots, X_{n}\right]$ where $n>1$. The elements $X_{1}, \ldots, X_{n}$ are of the principal class, so that $A=\left(X_{1}, \ldots, X_{n}\right)$ is basic. Lemma 2.5 shows that $A^{n}$ is not basic. We also note that each maximal ideal of $R$ is basic since each such ideal is of the principal class [9, Theorems 20 and 24, pp. 194 and 197]. Thus the condition that each maximal ideal of a ring $R$ be basic is not sufficient to conclude that $R$ has the basic ideal property.

3. Some results on basic ideals. Two of the goals of this paper are to characterize basic ideals and rings which have the basic ideal property. We have already given, in Lemma 2.2 and Theorem 2.3, two sufficient conditions in order that an ideal be basic. We now consider two simple results illustrating the usefulness of Lemma 1.3 .

An almost Dedekind domain is defined by Gilmer [4, p. 408] as a domain $D$ such that $D_{M}$ is Dedekind for each maximal ideal $M$.

Proposition 3.1. An almost Dedekind domain has the basic ideal property.

Proof. Observe that in each $D_{M}$ every ideal is invertible and thus basic. The result then follows by Lemma 2.2. Alternately, Proposition 3.1 follows from the fact that each nonzero ideal of an almost Dedekind domain is a cancellation ideal [4, p. 413].

Proposition 3.2. Let $A$ be an ideal of the ring $R$ which is contained in only one maximal ideal M. Then $A$ is basic if and only if $A^{e}$ is basic.

Proof. Assume that $A^{e M}$ is basic; then $A$ is basic by Lemma 2.2. Now let $B^{\prime}$ be a reduction of $A^{e M}$. Since $B=\left(B^{\prime}\right)^{c}$ contains a power of $A$, it follows from Lemma 1.3 that $B$ is a reduction of $A$. But $A$ is basic, hence $B=A$ and so $B^{\prime}=A^{e}$.

We observed in the last section that the product of basic ideals need not be basic; however, the next two results provide sufficient conditions for this to occur.

Theorem 3.3. The product of comaximal basic ideals is basic.

Proof. Let $A$ and $B$ be basic ideals such that $A+B=R$, and assume that $C$ is a reduction of $A B$, say $C(A B)^{n}=(A B)^{n+1}$. We claim that $C+A^{m}$ is a reduction of $A$ for each positive integer $m$. The ideal $C+A^{m}$ is contained in precisely those maximal ideals which contain $A$. Moreover, $\left(A^{n+1}\right)^{e}=\left((A B)^{n+1}\right)^{e}=$ $C^{e}\left((A B)^{n}\right)^{e}=C^{e}\left(A^{n}\right)^{e}$ for each such maximal ideal; and thus, by Lemma $1.3, C+$ $A^{m}$ is a reduction of $A$. We thus obtain $A=C+A^{m}$ for each positive integer $m$, and by a symmetric argument we show that $B=C+B^{n}$ for each positive integer $n$. 
After observing that $A^{m}$ and $B^{n}$ are comaximal for positive integers $m$ and $n$, we note that $C=C A^{n}+C B=C A+C B^{n}$. Therefore, $C B^{n}=C A^{n} B^{n}+C B^{n+1}=$ $\left(A^{n+1}+C\right) B^{n+1}=A B^{n+1}$. In a similar way, we obtain $C A^{n}=B A^{n+1}$, and from these two equations it follows that $C=C\left(A^{n}+B^{n}\right)=B A^{n+1}+A B^{n+1}=$ $A B\left(B^{n}+A^{n}\right)=A B$. Thus $A B$ is basic.

Theorem 3.4. Let $A$ and $B$ be ideals of the ring $R$, and assume that $A$ is invertible. Then $A B$ is basic if and only if $B$ is basic.

Proof. Let $B$ be basic and assume that $C$ is a reduction of $A B$, say $C(A B)^{n}$ $=(A B)^{n+1}$. Since $A$ is invertible, this implies that $C B^{n}=A B^{n+1}$. We note that $C A^{-1} \subset B$ and $C A^{-1} B^{n}=B^{n+1}$. Since $B$ is basic, $B=C A^{-1}$ and thus $C=A B$.

Now let $A B$ be basic and let $C$ be a reduction of $B$, say $C B^{n}=B^{n+1}$. We note that this implies that $C A \subseteq A B$ and $(C A)(A B)^{n}=(A B)^{n+1}$, and therefore $C A=B A$ since $A B$ is basic. Thus we obtain $C=B$ since $A$ is invertible.

The next example shows the somewhat surprising result that the extension of a basic ideal to a quotient ring need not be basic.

Example 3.5. Let $R=k[[X, Y, W, Z]]$ be the power series ring in four indeterminates over a field $k$. Then $R$ is a local ring and $X, Y, W, Z$ are analytically independent. We note that the ideal $\left(Z X^{2}, W X Y, Z Y^{2}\right)$ is basic by Theorem 2.3, as it is generated by analytically independent elements. And yet, if $P=(X, Y)$, then $A \subset P$, and $A R_{P}=\left(Z X^{2}, W X Y, Z Y^{2}\right) R_{P}=\left(X^{2}, X Y, Y^{2}\right) R_{P}$, which is not basic.

The following theorem seems to provide the best characterization of basic ideals in a Noetherian ring, in that it reduces the problem to that of basic ideals in local rings. By the results of [6], it is possible to determine the basic ideals in local rings with infinite residue field.

Theorem 3.6. An ideal $A$ in a Noetberian ring $R$ is basic if and only if $A^{e} \lambda$ is basic for eacb maximal ideal $M_{\lambda}$ containing $A$.

Proof. Lemma 2.2 shows that $A$ is basic if each $A^{{ }^{e} \lambda}$ is basic. Now let $A$ be basic and let $B^{\prime}$ be a reduction of $A R_{M}$, where $M \in\left\{M_{\lambda}\right\}$. We let $\left(B^{\prime}\right)^{c}=B$ and then show that $(B \cap A)+A M$ is a reduction of $A$. For $M_{\lambda} \neq M$, we observe that $[(B \cap A)+A M]^{e} \lambda=A^{e} \lambda$, and in $R_{M}$ the extension is $[(B \cap A)+A M]^{e}=B^{e}+$ $A^{e} M^{e}=B^{\prime}+A^{e} M^{e}$. The set of maximal ideals that contain $[(B \cap A)+A M]$ is precisely $\left\{M_{\lambda}\right\}$. Moreover, since $B^{\prime}\left(A^{e}\right)^{n}=\left(A^{e}\right)^{n+1}$, the relations obtained above in conjunction with Lemma 1.3 imply that $[(B \cap A)+A M] A^{n}=A^{n+1}$. Thus $A=$ $(B \cap A)+A M$ since $A$ is basic, and thus $A^{e}=B^{e}+A^{e} M^{e}$. Since $R_{M}$ is a local ring, it follows by [6, Lemma 1, p. 147] that $A^{e}=B^{e}$ and thus $A R_{M_{\lambda}}$ is basic for each $\lambda$. 
Corollary 3.7. Let $A \subseteq M$, a maximal ideal in a ring $R$, not necessarily Noetherian. If $B$ is an ideal of $R$ such that $B R_{M}$ is a reduction of $A R_{M}$, then $(B \cap$ $A)+A M$ is a reduction of $A$.

Proof. This is contained in the proof of Theorem 3.6.

Corollary 3.8. If $R$ is a Noetberian ring and if $B \cdot$ is a regular reduction of $A$, then $B$ is a minimal reduction of $A$ if and only if $B R_{M_{\lambda}}$ is a minimal reduction of $A R_{M_{\lambda}}$ for each maximal ideal $M_{\lambda}$ containing $A$.

Proof. We note that Theorem 1.2(b), implies that $B$ is a minimal reduction if and only if $B$ is basic, and this occurs if and only if $B R_{M_{\lambda}}$ is basic for each $\lambda$. But $B R_{M_{\lambda}}$ is basic if and only if it is a minimal reduction.

4. A special condition. It is easy to find examples in $k[X, Y]$, for instance Example 3.5, to show that there is no strong connection between an ideal and its primary decomposition with respect to the property of being basic. In this section, we consider ideals for which such a connection exists.

Theorem 4.1. Let $A$ be an ideal in a Noetberian ring $R$ sucb that each associated prime ideal of $A$ is maximal. An ideal $B$ is a reduction of $A$ if and only if $\operatorname{rad} B=\operatorname{rad} A$ and each primary component of $B$ is a reduction of the corresponding primary component of $A$.

Proof. Let $\operatorname{rad} B=\operatorname{rad} A$ and let each primary component of $B$ be a reduction of the corresponding primary component of $A$. If $B=\bigcap_{i=1}^{n} B_{i}$ and $A=\bigcap_{i=1}^{n} A_{i}$ are primary decompositions of $B$ and $A$, then we can choose $t$ so that $B_{i} A_{i}^{t}=$ $A_{i}^{t+1}$ for $i=1, \cdots, n$. It then follows from Lemma $1: 3$ that $B$ is a reduction of $A$.

Now suppose that $B$ is a reduction of $A$. Since $B$ contains a power of $A$, $\operatorname{rad} A=\operatorname{rad} B$ and it follows trivially that the components of $B$ are reductions of the corresponding components of $A$.

Corollary 4.2. Let $A$ be an ideal as described in Theorem 4.1; then $A$ is basic if and only if each primary component of $A$ is basic.

Proof. This is obvious by the theorem and the uniqueness of the primary decomposition of $A$.

Corollary 4.3. If $A$ is a regular ideal as described in Theorem 4.1, then $A$ bas a minimal reduction.

Proof. Let $P_{1}, \ldots, P_{n}$ be the associated prime ideals of $A$. Each $A^{e_{i}}$ has a minimal reduction $B_{i}{ }_{i}\left[6\right.$, Theorem 1, p. 147], and if we let $B=\bigcap_{i=1}^{n} B_{i}$, we can use Lemma 1.3 to show that $B$ is a reduction of $A$. That $B$ is a minimal reduction follows from Corollary 3.8 . 
Example 6.8 will show that an ideal may not have a minimal reduction; however, we may note that in a one-dimensional Noetherian integral domain, every ideal has a minimal reduction.

Theorem 4.4. Let $R$ be a Noetherian integral domain sucb that $R / M$ is infinite for every maximal ideal M. Eacb nonzero ideal bas an invertible reduction if and only if the dimension of $R$ is $\leq 1$.

Proof. We may assume throughout the proof that the dimension is nonzero, for if not, the result holds trivially. Assume that each proper ideal has an invertible reduction. Now let $P$ be a maximal prime ideal with invertible reduction $B$. Thus $B R_{P}$ is principal and so the height of $B R_{P}$ is 1 by the principal ideal theorem. This implies that height $P=$ height $P R_{P}=1$ and therefore the dimension of $R$ is $\leq 1$.

Conversely, if $\operatorname{dim} R=1$, let $A=\bigcap_{i=1}^{n} A_{i}$, where $A_{i}$ is $P_{i}$-primary. The condition that $R_{M}$ be infinite implies that the results of [6] hold for the quotient rings $R_{P_{i}}$. Each $A^{e_{i}}$ has a principal reduction $B_{i}^{e_{i}}$ [6, Theorem 1, p. 154], and if we form $B$ as in Corollary 4.3, then $B$ is an invertible reduction of $A$.

Corollary 4.5. Let $R$ be a Noetherian integral domain, not a field, such that $R / M$ is infinite for every maximal ideal $M$ of $R$. Then the dimension of $R$ is 1 if and only if every basic ideal is invertible.

Proof. If $R$ is one dimensional, Theorem 4.4 implies that every basic ideal is invertible. Conversely, if every basic ideal $A$ is invertible, then $A^{n}$ is basic for each positive integer $n$. It then follows by Theorem 2.7 that dimension $R=1$.

5. $C$-ideals. In this section we consider a notion dual to that of a basic ideal.

Definition 5.1. An ideal is a $C$-ideal if it not a reduction of any larger ideal.

Let $B$ be an ideal in a ring $R$ and let $x$ be an element of $R$ such that $x^{n}+$ $b_{1} x^{n-1}+\cdots+b_{n}=0$, where $b_{i} \in B^{i}$; then $x$ is integrally dependent on $B$. We note that if $B$ is a regular ideal of a Noetherian ring, $B$ is a $C$-ideal if and only if $B$ is integrally complete; that is, if $x$ is integrally dependent on $B$, then $x$ is in $B$. Moreover, we note that a $C$-ideal is integrally complete. To observe this fact, let $A$ be a $C$-ideal and let $x$ be integrally dependent on $A$. We immediately obtain the inclusion $\left(x^{n}\right) \subseteq A[A+(x)]^{n-1}$, which implies that $A$ is a reduction of $(A, x)$; thus $A$ is integrally complete. We show in the next section that the converse is false.

The following observations about $C$-ideals are immediate.

(1) A ring has the basic ideal property if and only if every ideal is a $C$-ideal.

(2) Prime ideals are $C$-ideals. 
(3) An ideal $B$ in a Noetherian ring is a $C$-ideal if and only if $B=\hat{B}$, where $\hat{B}$ is the largest ideal that has $B$ for a reduction.

Lemma 5.2. The intersection of any collection of $C$-ideals is a $C$-ideal.

Proof. Let $\left\{A_{\lambda}\right\}$ be a set of $C$-ideals and let $B=\bigcap_{\lambda} A_{\lambda}$. If $B \subseteq Q$ and

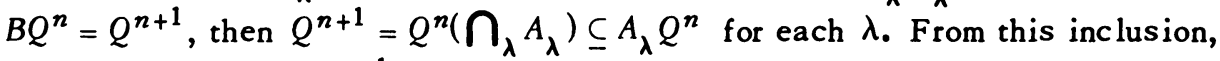
we note that $\left(Q+A_{\lambda}\right)^{n+1}=A_{\lambda}\left(Q+A_{\lambda}\right)^{n}$ for each $\lambda$. Since each $A_{\lambda}$ is a $C$-ideal, $Q \subseteq A_{\lambda}$ for each $\lambda$, and thus $Q \subseteq B$.

Theorem 5.3. Let $B$ be an ideal in a Noetherian ring and let $\left\{B_{\lambda}\right\}$ be the set of $C$-ideals which contain $B$; then $\hat{B}=\bigcap_{\lambda} B_{\lambda}$.

Proof. We first observe that $\hat{B}$ is a $C$-ideal. If not, there exists an ideal $C$ which has $\hat{B}$ for a reduction and we have $B \subseteq \hat{B} \subset C$; but this implies that $B$ is a reduction of $C[6$, Lemma 1 , p. 146], a contradiction to the definition of $\hat{B}$. We thus obtain the inclusion $B \subseteq \bigcap_{\lambda} B_{\lambda} \subseteq \hat{B}$, from which it follows easily that $\bigcap_{\lambda}$ is a reduction of $\hat{B}$. Thus by Lemma 5.2, $B_{\lambda}=\hat{B}$.

Corollary 5.4. Let $B \subseteq A$ be a regular ideal in a Noetherian ring; then $B$ is a reduction of $A$ if and only if $A$ is contained in eacb $C$-ideal containing $B$.

Proof. Apply Theorem 5.3 and Theorem 1.5.

Theorem 5.5. If $A$ is a regular ideal in a Noetberian ring, then $A$ is a $C$ ideal if and only if $[A Q: Q]=A$ for all regular ideals $Q$.

Proof. Let $A$ be a $C$-ideal and suppose that there exists a regular ideal $Q$ such that $A \subset[A Q: Q]$. After multiplying this proper inclusion by $Q$, we find that $A Q \subseteq[A Q: Q] Q \subseteq A Q$ and thus that $A Q=[A Q: Q] Q$. By [6, Theorem 2, p. 156], $A$ is a proper reduction of $[A Q: Q]$, a contradiction.

Now assume that $[A Q: Q]=A$ for each regular ideal $Q$ and let $A$ be a reduction of $B$, say $A B^{t}=B^{t+1}$. Upon noting that $B^{t}$ is a regular ideal, we see that $A \subseteq B \subseteq\left[A B^{t}: B^{t}\right]=A$. Thus $A$ is a $C$-ideal.

The next two results are analogous to Theorems 3.3 and 3.4 .

Theorem 5.6. If $A$ and $B$ are comaximal $C$-ideals, then $A B$ is a $C$-ideal.

Proof. Note that $A B=A \cap B$ and apply Lemma 5.2.

Theorem 5.7. Let $A$ be an ideal and let $B$ be an invertible $C$-ideal. Then $A B$ is a $C$-ideal if and only if $A$ is a $C$-ideal.

Proof. Let $A B$ be a $C$-ideal and assume that $A$ is a reduction of $Q$, say $A Q^{n}=Q^{n+1}$. After multiplying by $B^{n+1}$, we note that $A B$ is a reduction of $B Q$. Thus $A B=B Q$, which implies that $A=Q$. 
Now assume that $A$ is a $C$-ideal and that $A B \subseteq Q$ and $(A B) Q^{n}=Q^{n+1}$. We then note that $Q^{n+1} \subseteq B Q^{n} \cap A Q^{n}$ and this inclusion implies, as in Lemma 5.2, that $Q \subseteq A \cap B$. Since $B$ is invertible, there exists an ideal $F$ such that $B F=Q$. Substituting this into $A B Q^{n}=Q^{n+1}$, we see that $A B^{n+1} F^{n}=B^{n+1} F^{n+1}$.

Again using the invertibility of $B$, we note that $A F^{n}=F^{n+1}$ and that $A \subseteq F$. Thus $A=F$ and $Q=B F=B A$.

We observed in Example 3.5 that the extension of a basic ideal to a quotient ring need not be basic; however, the following theorem shows that the corresponding result for $C$-ideals is valid in the Noetherian case.

Theorem 5.8. Let $S$ be a multiplicative system in a Noetherian ring and let $B$ be a regular $C$-ideal such that $B \cap S=\varnothing$. Then $B R_{S}$ is a $C$-ideal.

Proof. If $B R_{S}$ is a reduction of $Q R_{S}$, let $\phi$ be the canonical homomorphism used in the formation of $R_{S}$ and let $x \in Q$. Then $\phi(x)$ is integrally dependent on $B R_{S}$. Thus there exist $b_{i}^{\prime} \in\left(B R_{S}\right)^{i}$ such that $\phi(x)^{n}+b_{1}^{\prime} \phi(x)^{n-1}+\cdots+b_{n}^{\prime}=0$. By clearing this equation of fractions, we see that there exist $n_{i} \in S$ such that $\phi\left(n_{0} x^{n}+n_{1} x^{n-1} b_{1}+\cdots+n_{n} b_{n}\right)=0$, where $b_{i} \in B^{i}$. By the definition of $\phi$, there exists $m \in S$ such that $m\left(n_{0} x^{n}+n_{1} x^{n-1} b_{1}+\cdots+n_{n} b_{n}\right)=0$. We then note that $m n_{0} x$ is integrally dependent on $B$ and thus is in $B$. Then $\phi(x)=\phi\left(m n_{0} x\right) / \phi\left(m n_{0}\right)$ $\epsilon B^{e}$ and so $Q R_{S} \subseteq B R_{S}$.

Theorem 5.9. Let $R$ and $S$ be as in Theorem 5.8 and let $B^{\prime}$ be a $C$-ideal in $R_{S}$; then $\left(B^{\prime}\right)^{c}$ is a $C$-ideal. $\left(B^{\prime}\right)^{c}$.

Proof. If $\left(B^{\prime}\right)^{c}$ is a reduction of $A$, then $B^{\prime}=A R_{S}$ and so $A \subseteq\left(A R_{S}\right)^{c}=$

Corollary 5.10. If $A$ is a regular ideal in a Noetherian ring, and if $\left\{M_{\lambda}\right\}$ is the family of maximal ideals containing $A$, then $A$ is a $C$-ideal if and only if $A^{e} \lambda$ is a $C$-ideal for eacb $\lambda$.

Proof. If $A$ is a $C$-ideal, then Theorem 5.8 implies that $A^{e} \lambda$ is a $C$-ideal for each $\lambda$. Now assume $A^{e} \lambda$ is a $C$-ideal for each $\lambda$; then apply Theorem 5.9 and Lemma 1.3 to complete the proof.

Theorem 5.11. In a Noetherian ring $R$, the following conditions are equivalent.

(1) $R$ is integrally closed in its total quotient ring $T$.

(2) Each invertible ideal is a $C$-ideal.

(3) Each regular principal ideal is a $C$-ideal.

Proof. $(1) \Rightarrow(3)$. Suppose that $(x)$ is regular and is not a $C$-ideal; this implies the existence of an element $y \notin(x)$ and elements $a_{i} \in R$ such that $y^{n}+a_{1} y^{n-1} x$ $+\cdots+a_{n} x^{n}=0$. Then there is an element $y / x$ not in $R$ which is integral over $R$. 
(3) $\Rightarrow(1)$. If an element $y / x \in T-R$ is integral over $R$, then, as above, $(x)$ is not a $C$-ideal.

$(2) \Leftrightarrow(3)$. That $(2) \Rightarrow(3)$ is clear. For the converse, let $A$ be invertible and a reduction of $Q$. Then there exists an ideal $B$ such that $A B=(x)$, where $x$ is regular. Clearly, $(x)=A B$ is a reduction of $Q B$, which implies that $A=Q$.

We note that the Noetherian property was used only in proving that (1) implies (3). We show in the next section that this hypothesis is necessary.

6. Results in Prüfer domains. In this section we show that although Prüfer domains do not have the basic ideal property, we can characterize them in terms of basic ideals. We first show a class of Prüfer domains which do have the basic ideal property.

Theorem 6.1. A 1-dimensional Prüfer domain D bas the basic ideal property.

Proof. Let $A$ be an ideal in $D$. If $A$ is idempotent and $B$ is a reduction of $A$, say $B A^{n}=A^{n+1}$, then $A=A^{n+1} \subseteq B$ and $A$ is basic.

If $A \neq A^{2}$ and $M$ is a maximal ideal containing $A$, then $A^{e}$ is an ideal in a valuation ring with $\operatorname{rad} A^{e}$ being the maximal ideal. We will show that such ideals are basic and then the theorem will follow from Lemma 2.2.

Let $B$ be an ideal in a valuation $\operatorname{ring}(V, M)$, such that $\operatorname{rad} B=M$. Suppose that $C$ is a proper reduction of $B$ and that $x \in B-C$. It then follows that $B^{2} C$ $C \subset(x) \subset B$ and thus that $(x)$ is a reduction of $B$. Since $(x)$ is invertible, there exists an ideal $Q$ such that $(x) Q=B^{2}$. We then observe that $(x)^{n} B^{n}=B^{2 n}=$ $(x)^{n} Q^{n}$ and this implies that $B^{n}=Q^{n}$; hence $B=Q$ [4, Lemma 20.4, p. 284].

If $y \in B-(x)$, then, as above, $(y)$ is a reduction of $B$ and $(y) B=B^{2}=(x) B$. Since $(x) \subset(y), x=r y$ for some $r \in M$ and this implies that $B=(r) B$, from which it follows that $B \subseteq B^{2}$, a contradiction.

We have been unable to determine the validity of the converse of Theorem 6.1, but we will show that Prüfer domains do not in general have the basic ideal property. We first need to establish the following result about prime ideals.

Theorem 6.2. A nonidempotent prime ideal $P$ of a valuation ring is principal or has a principal reduction.

Proof. Let $x \in P-P^{2}$; then we have $P^{2} \subset(x) \subseteq P$. Since $P^{2}$ is $P$-primary [4, Theorem 14.3, p. 173], it follows that $\left[P^{2}:(x)\right]=P$. We also have that $(x) Q=P^{2}$ since $(x)$ is invertible. Thus $P=[(x) Q:(x)]=Q$ and so $(x)$ is a reduction of $P$.

We will now consider an example of a Prüfer domain that does not have the basic ideal property.

Example 6.3. Let $V$ be a discrete valuation ring of rank two. Let $(0) \subset P \subset M$ be the prime ideals of $V$. We note that $P \neq P^{2}$ and that $P$ is not finitely generated. It follows from Theorem 6.2 that if $x \in P-P^{2}$, then $(x)$ is a proper reduction of $P$. 
The ideal $P$ does not have a minimal reduction. To see this, suppose that $B$ is a minimal reduction of $P$; we then have $P^{2} \subset B \subset P$. Let $x \in B-P^{2}$, then $(x)$ is a reduction of $P$ and so by the minimality of $B,(x)=B$. Now let $y \in M-P$; then $P^{2} \subset(y x) \subset(x)$ and again it follows that $(y x)$ is a reduction of $P$ which is strictly contained in the minimal reduction $(x)$, a contradiction.

We also note that we have $(y x) \subset(x) \subset P$ and $(y x)$ a reduction of $P$. However, $(y x)$ is not a reduction of $(x)$. Theorem 1.5 implies this could not happen in the Noetherian case.

Since a valuation ring is integrally closed, the ideal $(x)$ is integrally complete [4, Lemma 20.6, p. 287]; however, $(x)$ is not a $C$-ideal.

We will now describe Prüfer domains in terms of basic ideals. The condition of the following lemma will be the characterization.

Lemma 6.4. Assume that each finitely generated ideal of the integral domain $D$ is basic. Then $D$ is integrally closed.

Proof. We show that if $x / y \in F$, the quotient field of $D$, is integral over $D$, then $x / y \in D$. There exist $c_{i} \in D, i=1, \cdots, n$, such that $(x / y)^{n}+c_{1}(x / y)^{n-1}+$ $\cdots+c_{n}=0$. We note that $x^{n} \in(x, y)^{n-1}(y)$ and so $(y)$ is a reduction of $(x, y)$. By hypothesis $(x, y)$ is basic; therefore, $r y=x$ for some $r \in D$ and so $x / y=r \in D$.

Theorem 6.5. An integral domain $D$ is Prüfer if and only if every finitely generated ideal is basic.

Proof. It is clear that the condition is necessary. Assume that each finitely generated ideal is basic. It suffices to show that $D$ is integrally closed and that there is a positive integer $n>1$ such that $(a, b)^{n}=\left(a^{n}, b^{n}\right)$ for all $a, b \in D$ [4, Theorem 20.3, p. 281].

We have that $D$ is integrally closed by Lemma 6.9; and for every $a, b \in D$, $(a, b)^{2}=\left(a^{2}, b^{2}\right)$ since $(a, b)^{2}$ is basic. Therefore $D$ is Prüfer.

Corollary 6.6. A Noetherian domain is Dedekind if and only if it has the basic ideal property.

\section{BIBLIOGRAPHY}

1. H. S. Butts and R. Gilmer, Primary ideals and prime power ideals, Canad. J. Math. 18 (1966), 1183-1195. MR 37 \#204.

2. E. D. Davis, Ideals of the principal class, $R$-sequences and a certain monoidal transformation, Pacific J. Math. 20 (1967), 197-205. MR 34 \#5860.

3. - Further remarks on ideals of the principal class, Pacific J. Math. 27 (1968), 49-51. MR 39 \#191.

4. R. Gilmer, Multiplicative ideal theory, Queen's Papers in Pure and Appl. Math., no. 12, Queen's University, Kingston, Ont., 1968. MR 37 \#5198. 
5. R. Gilmer and W. Heinzer, On the number of generators of an invertible ideal, J. Algebra 14 (1970), 139-151. MR 40 \#5598.

6. D. G. Northcott and D. Rees, Reductions of ideals in local rings, Proc. Cambridge Philos. Soc. 50 (1954), 145-158. MR 15, 596.

7. - A note on reductions of ideals with an application to the generalized Hilbert function, Proc. Cambridge Philos. Soc. 50 (1954), 353-359. MR 15, 929.

8. O. Zariski and P. Samuel, Commutative algebra. Vol. 1, University Series in Higher Math., Van Nostrand, Princeton, N. J., 1958. MR 19, 833.

9. - Commutative algebra. Vol. 2, University Series in Higher Math., Van Nostrand, Princeton, N. J., 1960. MR $22 \# 11006$.

DEPARTMENT OF MATHEMATICS, NORTHEAST MISSOURI STATE COLLEGE, KIRKSVILLE, MISSOURI 63501 